Journal of Engineering and Applied Sciences 14 (Special Issue 6): 9245-9250, 2019

ISSN: 1816-949X

(C) Medwell Journals, 2019

\title{
The Interpretation of Dyslexic Children's Facial Expressions While Using a Reading Courseware
}

\author{
${ }^{1}$ Fakhrul Anuar Aziz and ${ }^{1}$ Zulikha Jamaludin \\ ${ }^{1}$ School of Multimedia Technology and Communication, \\ ${ }^{2}$ School of Computing, Universiti Utara Malaysia (UUM), 06010 Sintok, Kedah, Malaysia
}

\begin{abstract}
A reading courseware prototype, MyBaca is built for the purpose of assisting dyslexic children to improve their reading skills. MyBaca is an interactive application which is designed based on Affective Engineering (AE) Model and the needs and reading patterns of dyslexic children. The prototype has gone through the development and implementation phase. Hence, there is a need to evaluate MyBaca by looking at emotional responses from the users as it has never been evaluated before. Hence, the objective of this study is to evaluate the AE Model translated into the prototype in terms of its affective dimensions. This study presents a translation of facial expressions of the users into affective impressions while they are using the prototype. Observation has been performed using video recordings to record facial expressions and point of interactions of the users and the results are recorded in a printed form based on LEM tool. The results show that the users portrayed positive affective states while using the prototype hence confirming to the AE Model translated into the prototype.
\end{abstract}

Key words: Facial expression, affective quality, affective dimension, reading courseware, prototype, dyslexic children, animated pencil

\section{INTRODUCTION}

Recognizing and interpreting facial expressions is a key part of human-to-human communication. It is essential in research especially when involving affective qualities in a product. The study of interactive product design based on emotion stressed that research related to emotion triggered ideas in the field of human computer interaction and it is important to consider aspects of user's emotion in design and evaluation processes. The study also added that affective dimension contributes to the overall design goal.

In parallel with the importance of emotional based research in design, we developed MyBaca a reading application prototype for Malay language where it considers affective dimension Interaction Design $(\mathrm{I} \times \mathrm{D})$ and impairments of Dyslexic Children (DC) spesifically on their reading patterns. The research on MyBaca was motivated by the need to offer DC with an effective, engaging and fun learning process. The main objective of the research is to create an affective engineering model for $\mathrm{I} \times \mathrm{D}$ in order to supports the reading pattern of $\mathrm{DC}$.

The problem in this research is that the model is still not evaluated in terms of the affective dimension of real users. Thus, there is a need to evaluate the prototype through subjective or emotional responses of the users. For the purpose of this research we choose facial expression as the method to elicit the user's affective dimensions. Since, MyBaca courseware is designed by following the proper affective engineering model, the DC would find it interesting and fun to use (Aziz et al., 2013). It also should motivate them to learn, engage and promote positive feelings when using it.

\section{MATERIALS AND METHODS}

Affective dimension: Based on psychological studies it was found that emotion emanates from the inside of the brain. Specifically, it started in the limbic system (Wujec, 2009; Marks and Nesse, 2001). The limbic system is a small structure located at the center of the brain as presented in Fig. 1 adopted from Wujec (2009). The role of lymbic system is to translate and trigger emotions and behaviors that will be sent to the brain cortex that controls logical or rational operations (Ekman, 2003).

The limbic system is a set of evolutionarily primitive brain structures located on top of the brainstem and buried under the cortex (Bailey, 2015). Limbic system structures are involved in many of our emotions and motivations, particularly those that are related to survival.

Corresponding Author: Fakhrul Anuar Aziz, School of Multimedia Technology and Communication, Universiti Utara Malaysia (UUM), 06010 Sintok, Kedah, Malaysia 


\section{J. Eng. Applied Sci., 14 (Special Issue 6): 9245-9250, 2019}

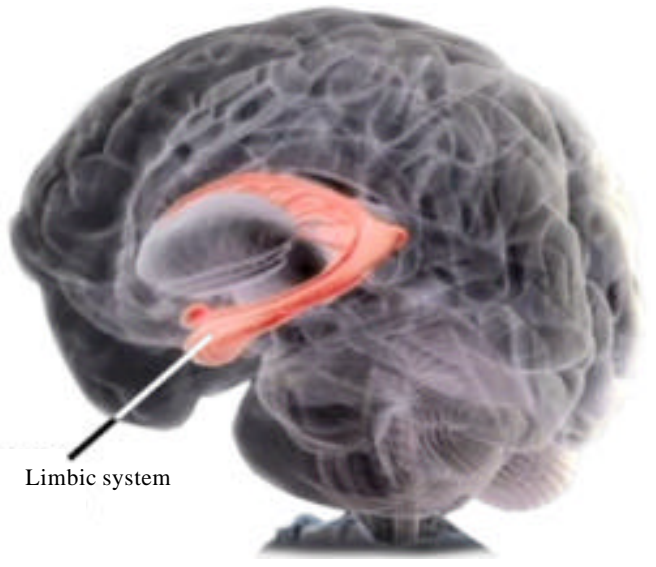

Fig. 1: The location of limbic system inside human brain (Aziz et al., 2013)

Such emotions include fear, anger and emotions related to sexual behavior. The limbic system is also involved in feelings of pleasure that are related to our survival such as those experienced from eating and sex.

Feelings are triggered when people see an object or experiencing an event (Lokman and Nagamachi, 2010). Accordingly, the limbic system affects human more than the cortex (Cytowic, 1996). Hence, the finding supports the idea that affective aspects in human are important to be considered especially in human computer interaction.

Targeting at arriving to user's affective impressions, Fig. 2 shows the core affect circle that shows two dimensions of neuro-psychological states: pleasure-displeasure state and activated-non-activated state (Russell, 2003). Based on the two dimensions, another dimension can be plotted that shows positive affect at one end and negative affect at the other. Hence, in digital product design we target that the application should generates positive affect in the users mind, so that, they are happy with or engaged in such application.

Again, referring to the core affect as illustrated in Fig. 2, the reason why Affective Engineering (AE) is vital in product design is to elicit requirements that can create pleasure and activation and thus bring out positive affect when user uses a product. With this approach, a better user-appealed product can be designed. Hence, the product that has been designed using $\mathrm{AE}$ is supposed to move the emotional affective state of its user towards the positive affective state. When in this state, pleasure and activation are integrated into one creating an emotionally positive feeling (Aziz et al., 2013).

In digital product design, affective quality and affective impression should be taken as the vital aspects. Affective quality is the object's ability to cause a change in the user's core affect. Examples of the objects are $I \times D$

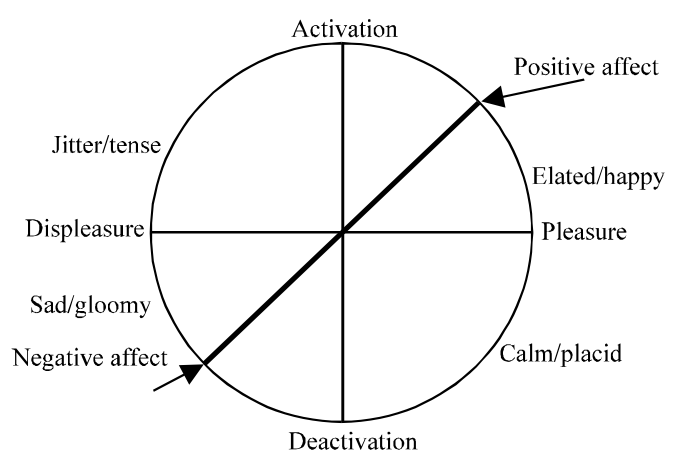

Fig. 2: Core affect circle (Cytowic, 1996)

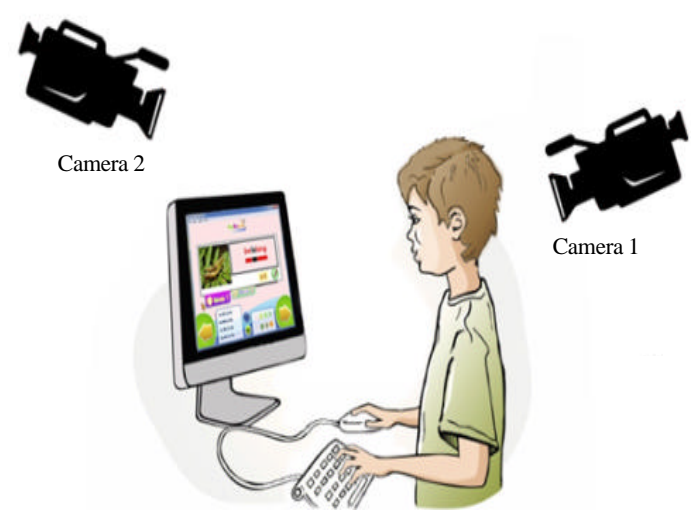

Fig. 3: Video recording using two cameras

components in the courseware or application such as colour, image, text, animation, audio and video. On the other hand, affective impression is the user's appraisal of the affective objects in $\mathrm{I} \times \mathrm{D}$ components such as sadness, tense, happy and calm (Te'eni et al., 2007; Nagamachi, 2008).

Video recording: This study is aimed at discovering the design impact of the stimuli (i.e., MyBaca) to the emotional response (affective dimension) of the user. The features of MyBaca includes the main menus, navigation buttons, pencil animation, word colour, word display, animated pencil, cursor movement, audio, pictures and most importantly flexible choices of font types, font sizes and eight background colours. For the purpose of this study, only three functions in MyBaca courseware have been evaluated. They are Word Colour (WC), Word Display (WD) and Animated Pencil (AP).

WC consists of different coloured syallables where the default is offered in black and red. However, DC can customize the syllable colours according to their preferences. WC is designed based on the fact that DC is affected by words and its background colours. Different 
colour helps them to differentiate the syllables in a particular word. WD is the display of a word on the screen. DC can choose the font type and the font size according to their preferences in order to read a word. Normally, DC would choose the type and size of font that make them feel comfortable to read. These features help them to configure the content (word) to fulfill their needs.

$\mathrm{AP}$ is an animation of a pencil illustating how to write an alphabet in slow motion. It gives a clue to DC of the starting point and the direction to start reading a word. Furthermore, it helps DC to remember how an alphabet is shaped, crafted and to create individual sounds and combine them to pronounce a word.

Meanwhile, the choosen method to capture emotion of the users is through video recording. We elaborate the settings of video recording while user is interacting with the prototype in two parts 1 the camera setup and 2 the video mixing technique.

Camera setup: Video recording has advantage that we can see what the participant or user is doing and reacting. Hence, in order to record them, the setting is established as shown in Fig. 3. There are two cameras camera: 1 and 2. Camera 1 is set behind the user and intentionally to record the computer screen. Camera 2 is located infront of the user and used to record the facial expressions of the user. It is suggested the the cameras are attached on tripods in order to gain stable video images. The camera is switch on to recording mode a couple of min before the user interacting with the application, so that, we are not missing the crucial data of user's facial interface while they started the session.

Video mixing: After completing the recording part, the two videos are mixed as one movie clip. They are alligned in a screen, so that, both can be viewed at once as shown in Fig. 4. We mixed the two videos (for each user) by using Adobe Premier CS6 video editing software. The still image snap function is also provided in the workspace, so that, significant facial expressions on the targeted task can be captured easily for reporting.

Observation: The observation method is used in order to capture the facial expressions of the users especially for special needs children as suggested in Fig. 4: two videos mixed as a single movie clip in order to map user task and the facial expression: "Observation is as important as testing and much can be revealed by how a child approaches tasks within the classroom for example, how

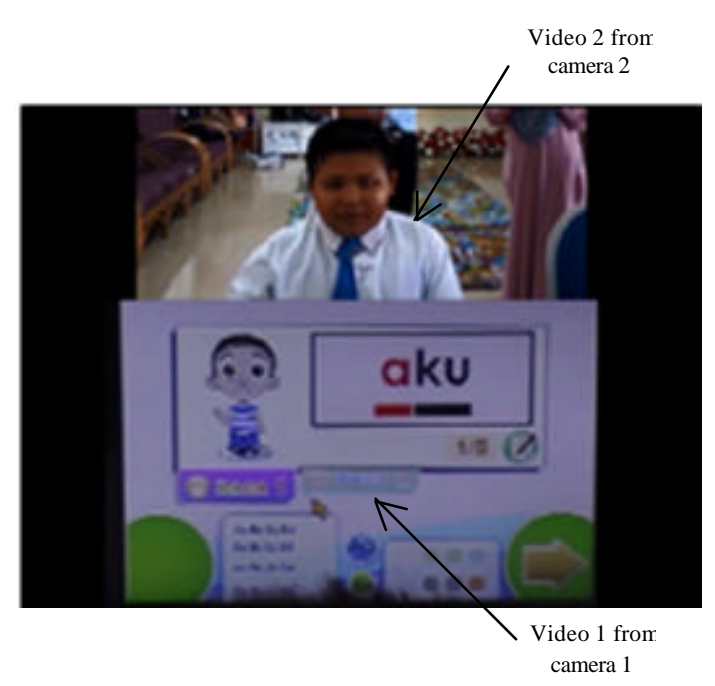

Fig. 4: Two videos mixed as a single movie clip in order to map user task and the facial expression

they cope with uncertainty, whether they seek help, if they have the endurance to see tasks through to completion or whether they utilise a bank of strategies". Observation is used as the method to gather useful information in design process as it is very practical and easy to get children participate in the study (Hourcade, 2015).

In this study, the main technique used in observation is cooperative inquiry (Hourcade, 2015) using high fidelity prototype of MyBaca to perform video recording of the children using the prototype in order to capture facial expressions of the users as We observed 10 dyslexic children in primary school, whose age ranges from 9-11 years old. Although, these children are dyslexics with special needs when it comes to learning to read, their cognitive ability is at par with their normal peers (Shaywitz, 2008). Since, MyBaca contains different difficulty levels, we, thus, need to include children from various range of reading levels and the reading levels are identified by the teachers who are well acquainted with the children's performance in school. The main thing to consider is to get them engage in the process so that they can participate well during the video recording. However, since, using computers is just engaging and fun enough for children (Hourcade, 2015), the process thus went well.

Firstly, we recorded their interactions with the reading prototype, MyBaca. Then, we processed the recorded videos and then trancribed them by using facial expression guide which is adopted from LEMtool. 
Table 1: Affective dimension of facial expressions

Affective dimension

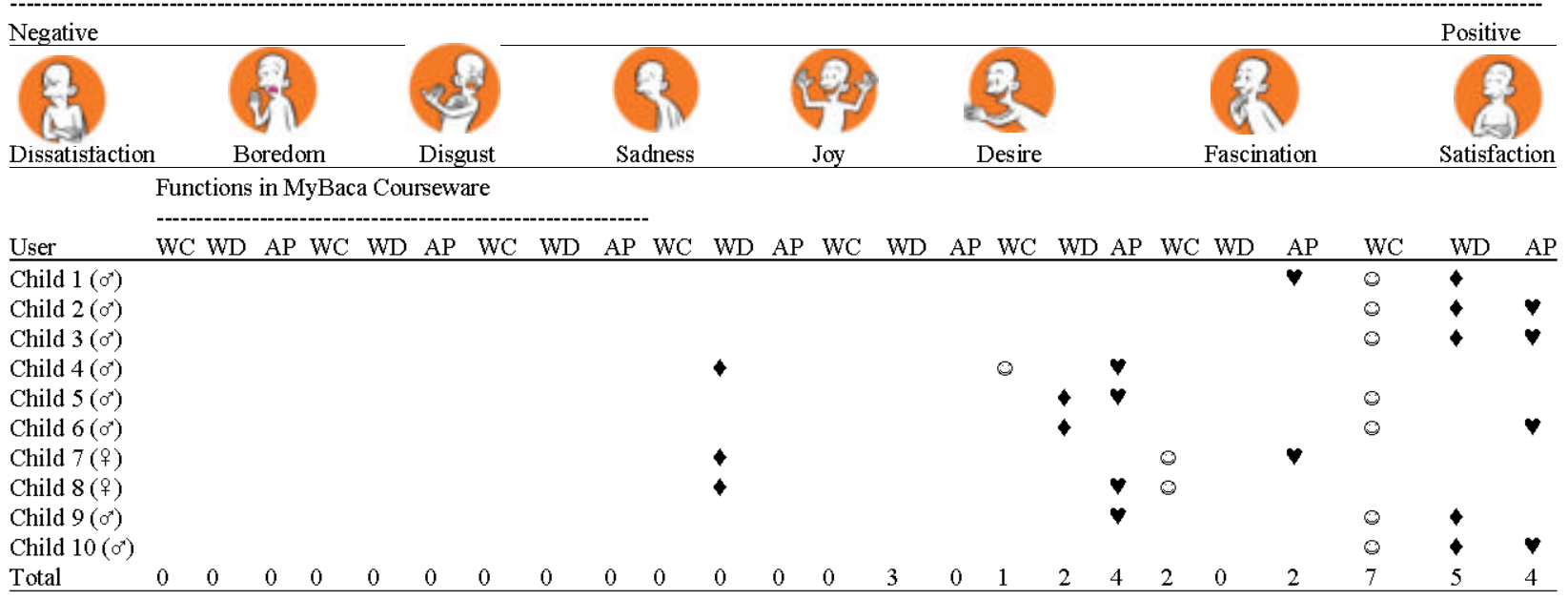

An emotion symbol for WC; for WD and $\mathbf{\psi}$; For AP

LEMtool consists of eight validated emotions. The positive emotional responses are joy, desire, fascination and satisfaction whilst the negative expressions are dissatisfaction, boredom, disgust and sadness. LEMtool is easy to use and very reliable. The original LEMtool is an online tool to measure web-based application and media including sketches, mock-ups, early design and any other images. This tool makes it possible to uncover the user's emotional impact of design of any digital product (Aboagye, 2015).

The components inside LEMtool such as emoticons, characters and tool have been scientifically validated (Huisman et al., 2013). Since, it is claimed to be proven and reliable, we adopted the LEMtool cartoon characters as a guide in a printed form in order to gain insight into user's affective impressions (or emotional responses) towards MyBaca. In other words, the interface of MyBaca acts as the stimuli during the observation and the affective responses are recorded in the printed form based on LEM tool.

Translation process: While the children are using the prototype of MyBaca, their expressions are continuously observed. The translation process of user's facial expressions into affective dimension data involves matching the expressions observed with eight existing emotion characters based on LEMtool.

For the purpose of this study, three functions in MyBaca courseware have been evaluated. They are WC, WD and AP as shown in Table 1. The observer will ticks the most approriate emotional responses of the user based on LEMtool's facial expressions guideline. For example if the user smiles at the targeted interaction point, the "Satisfaction icon" will be ticked. Otherwise if the user frowns and shows a sad face, the "Sad icon" will be chosen. The expression recorded is the first impression shown by the children while using specific target features to be observed. There can also be a situation where a child did not really portray any facial expression that can be addressed by the eight emotion characters. Hence, his/her body language need to be observed too prior to capture his/her affection towards the specific functions in MyBaca (Lera and Domingo, 2007).

\section{RESULTS AND DISCUSSION}

The user's facial expressions data while interacting with targeted interaction points are shown in Fig. 5 whilst Table 1 is presenting the result of the user's facial expressions data which translated into user's affective dimension.

From the affective dimension data as mentioned above, we can see that all of the users tend to show positive impressions while interacting with MyBaca courseware. In term of the features of MyBaca) WCshows that $70 \%$ of users satisfied, $20 \%$ fascinated and $10 \%$ desired) WD 50\% satisfied, 30\% joyed and 20\% desired) AP 40\% satisfied, 40\% desired and 20\% fascinated. The data apparently show that the WC, WD and AP are accepted by all of the users because they show positive impression on it. Hence, the design of MyBaca could help and motivate them to learn to read. 
(a)

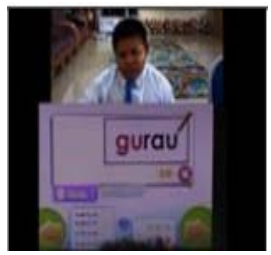

(f)

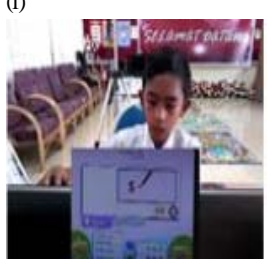

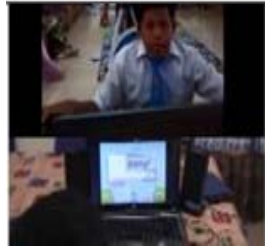

(g)

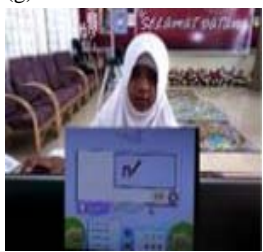

(c)

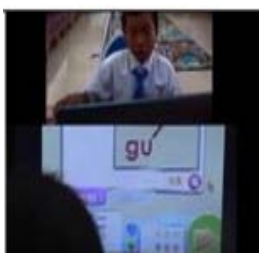

(h)

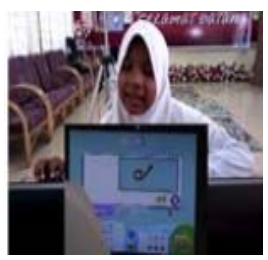

(d)

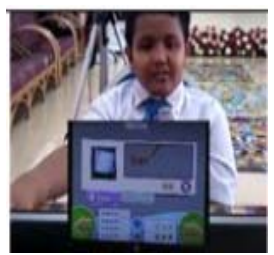

(i)

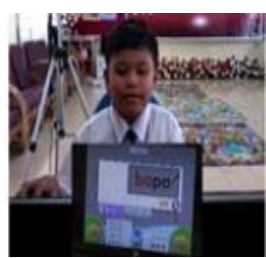

(e)

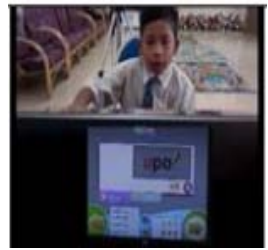

(j)

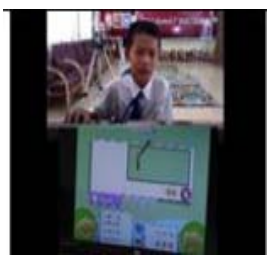

Fig. 5: Facial expressions of the user while interacting with animated pencil, word colour and word display: a) Child 1; b) Child 2; c) Child 3; d) Child 4; e) Child 5; f) Child 6; g) Child 7; h) Child 8; i) Child 9 and j) Child 10

\section{CONCLUSION}

The main point of the study is to highlight the process of observing and recording the facial expressions of users while they are interacting with a reading application prototype, i.e., MyBaca and translating the user's facial expressions into affective dimension. The importance of the work is to provide a way to evaluate a prototype in terms of subjective responses from target users. The results show that the prototype of MyBaca is acceptable by its target users as they, i.e., the DC, portray positive affective state while using MyBaca, i.e., joy, desire, fascination and satisfaction. None of the DC shows any negative state while using MyBaca. Hence, the results confirm that the design of MyBaca which is based on $\mathrm{AE}$ model is able to position the emotional affective state of DC towards achieving positive affective state.

According to the results, the three features of MyBaca that have been evaluated, i.e., WC, WD and $\mathrm{AP}$ show an interesting pattern that there are differences in the positive affective dimensions between boys and girls. From gender perspective, the girl's affective dimensions are joy and fascination while the boys are more towards satisfaction. This could potentially be, so, because dyslexia occurs mostly to boys rather than girls. The design of MyBaca is constructed from profiling of dyslexic children that are mostly boys (Aziz et al., 2008). Therefore, this design is satisfying for the boys. However, it does not indicate that the girls who are being evaluated did not like to use it as they too show the evidence of being positively stimulated when using MyBaca as demonstrated in the results and in Table 1.

\section{RECOMMENDATIONS}

For future researcher, all of the features in the reading application prototype will be evaluated based on user's affective impressions by using the same method. The other features that will be evaluated include the navigation buttons, choices of font types and font sizes, choices of background colours, pencil animation (for sentence level), cursor movement, audio, picture and word pronunciation activity.

\section{REFERENCES}

Aboagye, S., 2015. A unique approach to dyslexia. SEN Magazine, UK. https://senmagazine.co.uk/ articles/articles/senarticles/what-do-model-railwaysand-chocolate-tasting-have-to-do-with-teachingdyslexics

Aziz, F.A., H. Husni and Z. Jamaludin, 2008. Defeating phonological-core deficit among dyslexic children by activating arousal dimension of affective impressions. Proceedings of the International Conference on Education, Research and Innovation, November 1719, 2008, IATED, Madrid, Spain,ISBN:9788461250912, pp: 1-1.

Aziz, F.A., H. Husni and Z. Jamaludin, 2013. Translating interaction design guidelines for dyslexic children's reading application. Proceedings of the World Congress on Engineering, Vol. 2, July 3-5, 2013, Newswood Limited, London, England, UK., ISBN:978-988-19252-8-2, pp: 1-4.

Bailey, R., 2015. Limbic system. About, Inc, New York, USA. http://biology.about.com/od/ anatomy/a/aa042205a.htm. 
Cytowic, R.E., 1996. The Neurological Side of Neuropsychology. MIT Press, Cambridge, Massachusetts, USA., Pages: 486.

Ekman, P., 2003. Emotions Revealed: Understanding Faces and Feelings. Orion Publishing Group, London, England, UK.,

Hourcade, J.P., 2015. Child-Computer Interaction. APE Author Publisher, Iowa City, Iowa, USA.,.

Huisman, G., V.M. Hout, V.E. Dijk, V.D.T. Geest andD. Heylen, 2013. LEMtool: Measuring emotions in visual interfaces. Proceedings of the SIGCHI Conference on Human Factors in Computing Systems, April 27-May 2, 2013, ACM, Paris, France, ISBN:978-1-4503-1899-0, pp: $351-360$.

Lera, E.D. and G.M. Domingo, 2007. Ten emotion heuristics: Guidelines for assessing the user's affective dimension easily and cost-effectively. Proceedings of the 21 st British HCI Group Annual Conference on People and Computers: HCI... But not as we Know it, Vol. 2, September 3-7, 2007, British Computer Society, Swindon, England, UK., ISBN:978-1-902505-95-4, pp: 163-166.

Lokman, A.M. and M. Nagamachi, 2010. Kansei Engineering: A Beginners Perspective. University Pub. Centre (UPENA), Shah Alam, Malaysia, ISBN: 9789673631230 ,
Marks, M. and R.M. Nesse, 2001. Fear and Fitness: An Evolutionary Analysis of Anxiety Disoders. In: The Science of Mental Health: Fear and Anxiety, Hyman, S. (Ed.). Routledge, New York, USA., ISBN:0-81533753-1, pp: 155-169.

Nagamachi, M., 2008. Perspectives and the new trend of Kansei-affective engineering. TQM. J., 20: 290-298.

Russell, J.A., 2003. Core affect and the psychological construction of emotion. Psychol. Rev., 110: 145172.

Shaywitz, S., 2008. Overcoming Dyslexia: A New and Complete Science-Based Program for Reading Problems at any Level. Vintage Publisher, New York, USA.,

Te'eni, D., J.M. Carey and P. Zhang, 2007. HumanComputer Interaction: Developing Effective Organizational Information Systems. John Wiley \& Sons, Danver, Massachusetts, USA., ISBN:9780471677659, Pages: 436.

Wujec, T., 2009. Three ways the brain creates meaning. TED Conferences, LLC, Manhattan, New York, USA. https://www.ted.com/talks/tom_wujec_on_3_ways _the_brain_creates_meaning. 